\title{
The Study on Application of Probability and Statistics Mathematics on Investment Decision
}

\author{
Sheng Yuan ${ }^{1}$ \\ ${ }^{1}$ Yunnan Urban Construction Vocational College, Yunan, 651701 \\ 54892713@163.com
}

Keywords: Probability; Statistics Mathematic; Application Study

\begin{abstract}
To reduce the project risk, ensure the accuracy of investment decisions, from the perspective of economic decision-making and through an analysis of project investment decision potential risks, this paper build a set of fuzzy comprehensive evaluation models. For the project investment risk certainty fuzziness, this paper uses probabilistic model to various risks totality judge accurately realized the data from a static to a dynamic of change, the various difficult problems encountered within the economic range thinking by way of a mathematical model for to provide a scientific basis for investment decisions.
\end{abstract}

\section{Introduction}

Mathematics is the study of natural and social phenomena in the form of the number of relationships and space in a very broad sense science. As an important basic disciplines and a precise scientific language, mathematics and science it is an extremely abstract form appears. This form is sometimes very abstract mathematics will cover rich content, and may be an obstacle to the practical application of mathematics. Use mathematical methods to solve a practical problem, whether the problem is from the engineering, economic, financial or social sphere, must set up a bridge between the practical problems with mathematics, is the first real problem into a mathematical problem then this mathematical problem analysis and calculation, and finally the actual return obtained answer, whether we can effectively answer the original practical problems. This whole process is called mathematical modeling.

By building a mathematical model, the use of mathematical knowledge to solve practical problems, to promote our scientific and technological innovation, economic progress has positive significance. The main problem of probability theory to solve the problem is uncertainty. Mathematical statistics, probability theory is based on statistical inference problems. Probability theory and mathematical modeling of statistical methods to solve application problems in the economic field has a wide range of applications.

\section{Statistical Modeling Method}

Overview of Statistical Modeling. Statistical modeling based on statistical analysis computer software as a tool, using a variety of analytical methods for the process of establishing a statistical model and data processing, these results can be used directly in the efficiency of enterprises project forecast on project basis. At present, the statistical analysis statistical offices have been established with the data model and the application of statistical data to play a role in basic conditions.

Power, fuel and other five categories of products prices rose. 2003 to 2005, Inner Mongolia fuel, agricultural class, other industrial raw materials, chemical materials, textile raw materials and power classes and other five categories of products increase purchase prices for five years. Among them, chemical raw materials, fuel and power prices a larger category of products.

Textile raw materials, timber and other raw materials purchase prices relatively stable, volatility is not. From 2003 to 2007, the region's textile raw materials, timber and other industrial raw materials purchase prices relatively stable and accompanied by a rising trend, ups and downs is not great. 
Overall, the nine categories of raw material prices are mostly upward trend, from 2003 to 2007 the proportion of the region's raw materials price rises accounted for a relatively large decline in the proportion accounted for a relatively small, in 2004 and 2005 During the nine raw material price hikes, of which there are five categories of five consecutive years of rising raw material prices, the other four categories of the phenomenon appears to lower prices in individual years, but later remained upward trend.

According to the survey, five years, most of the product purchase prices are rising. In the survey of 37 industries, which, within five years the price of the entire industry rose 31 . In 2005, the purchase price of 37 industry products are rising, the rest of the year the industry product purchase prices still keep rising trend.

From 2003 to 2007 the region's non-ferrous material, ferrous metal material, and chemical raw materials purchasing price fluctuations more obvious, these three categories of products are purchase prices showed a substantial upward trend in 2004, while ferrous metal materials beginning in 2005 decline, until 2007, the price rise. Chemical raw materials purchase prices of products during the five-year fluctuations more obvious. Purchase prices of agricultural products continued to rise within five years, in 2004 the five-year record high. Other years also are up and down, but the overall upward trend.

Practice in Economic Management Decision. Before economic management decisions, usually there will be uncertainty, randomness, therefore, there is a certain risk decision made, only correct and reasonable decision sciences in order to achieve the minimum cost to reap the greatest benefit of the overall goal, as far as possible in order to save investment costs. In order to achieve the ultimate goal by using knowledge of probability and statistics to develop a reasonable decision the following mathematical expectation, variance, etc. It is calculated as an example in economic management decision.

Example 1 a person has a fund, invested a total of three projects: Property A, Property B and commercial C, its earnings and market conditions, and if the future market is divided into excellent, good, poor three levels, the probability of its occurrence respectively, $\mathrm{P} 1=0.3, \mathrm{P} 2=0.0 .6, \mathrm{P} 3=0.1$, depending on the circumstances of market research shows that annual earnings under all kinds of different levels of state investment, are shown in Table 1: excellent $\mathrm{P} 1=0.3$ good $\mathrm{P} 2=0.6$ difference P3 = 0.1 Commercial Property 53-1.92-3.103-4 estate. How the investors should invest?

Solution: It can be seen: $\mathrm{E}: \mathrm{A})=10 * 0.3+3 * 0.6+:-4) * 0.1=4.4 ; \mathrm{E}: \mathrm{B})=5 * 0.3+3 * 0.6+:-1)$ $* 0.1=3.2 ; \mathrm{E}: \mathrm{C})=9 * 0.3+2 * 0.6+:-3) * 0.1=3.6$; according to the above algorithm shows that the average income of the largest real estate investment, may choose to real estate, but the risk of the investment is relatively large, we then from the variance be considered: :A)=:10-4.4) $2 * 0.3+: 3-4.4) 2 * 0.6+:-4-4.4) 2 * 0.1=17.14 \mathrm{D}: \mathrm{B})=: 5-3.2) 2 * 0.3+: 3-3.2) 2 * 0.6+$ $:-1-3.2) 2 * 0.1=2.76 \mathrm{D}: \mathrm{C})=: 9-3.6) 2 * 0.3+: 2-3.6) 2 * 0.6+:-3-3.6) 2 * 0.1=14.64$.

Statistical models have a more mature stage of applicable tools, master a large statistical resources, on this basis, the application of statistical work in statistical modeling to solve management problems has become a trend. With the development of the field of statistical data validation, calibration methods have accurate information as a basis for statistical work prospects will be bright. The principle of the planned investment is ongoing reform, who invests, who benefits and who bears the risk, to make investment decisions to become more autonomous. At the same time, improve the investment macro-control system, strengthen supervision is imperative. Therefore, the implementation of statistical models becomes particularly important.

\section{Establish Parameters Selection Model}

Establish general statistical model in three phases: analysis have mastered the hands of a variety of information; determining a variety of specific issues need to be addressed, the selection of appropriate indicators of the way to solve the problem; to establish an effective working model of experimental mathematics.

Mainly through a variety of existing statistical information on the various information resources to do an effective combination, there are plans to establish a mathematical model of the project and 
rules of decision analysis, more in-depth and detailed statistics play out the functions, therefore, establishment of a planned decision to review the operation, the selection of a mathematical model to identify the problems to be solved.

Indicator model uses the more there are three kinds of ways, including the following three aspects. First, a single economic indicator models, as soon as the target for investment decisions only when the economic benefits of a simple process that can be done to calculate the index of each alternative economic program, and in accordance with specific economic indicators do effective investment return rate, Net finance etc., because it is only necessary to consider the various single goal to make the goal of this decision is relatively simple, it is also easier to operate, the main drawback is that the program can not be done on the overall thinking, the second, a comprehensive index model, when the investment decision-making process there are several alternative target, on the outcome of various factors and determine the need for various options to do a comprehensive analysis, multi-objective manner determined by the score includes the right way and so on, the weighted scoring mode, value analysis mode, grade analysis methods, and the center of the target multi-objective decision is to determine which factors are effective indicators, plays a decisive role, and make a comprehensive comparison after a comprehensive analysis, and determination of the result of the selection made, the first Third, the uncertainty of the model, a variety of uncertain decision model, the probability of the future needs to be a variety of natural conditions into consideration of, and some even take place in the form of natural circumstances; and in accordance with the diverse nature Evaluation forms and the probability of occurrence is determined to make decision-making non-probabilistic model of determination, breakeven analysis mode is not the model to calculate the probability of the uncertainty of the decision, in the form of various simulation model to calculate the probability is sporadic decision.

\section{Risk Analysis}

Investment decisions referring to the developers of the project through the project development before the country's macro factors, including the political, policy, economic, financial and market environment within a small range, the economic environment, the project itself block range, specific investment directions , investment, investment opportunities, and so do effective analysis, judgment, and by means of feasibility analysis, changes in supply and demand on the market environment, and so do the analysis in the form of price developments, effectively projected earnings within the determination to do the evaluation, and comprehensive Analysis of economic and environmental technologies, to develop the best model portfolios,

Investment decisions referring to the developers of the project through the project development before the country's macro factors, including the political, policy, economic, financial and market environment within a small range, the economic environment, the project itself block range, specific investment directions , investment, investment opportunities, and so do effective analysis, judgment, and by means of feasibility analysis, changes in supply and demand on the market environment, and so do the analysis in the form of price developments, effectively projected earnings within the determination to do the evaluation, and comprehensive Analysis of economic and environmental technologies, to develop the best model portfolios.

Investment decisions referring to the developers of the project through the project development before the country's macro factors, including the political, policy, economic, financial and market environment within a small range, the economic environment, the project itself block range, specific investment directions , investment, investment opportunities, and so do effective analysis, judgment, and by means of feasibility analysis, changes in supply and demand on the market environment, and so do the analysis in the form of price developments, effectively projected earnings within the determination to do the evaluation, and comprehensive Analysis of economic and environmental technologies, to develop the best model portfolios.

There are various investment projects decided uncertainty, the risk of a large presence in the development process, most of the uncertainty of the results of the project development factors will cause serious impact, so the decision is correct, direct, and the entire project development The 
success or failure are closely linked, where you can determine investment risks by means of a formula to do the analysis, a specific formula.

In the above formula, the parameter $\mathrm{J}$ represents the project investment decisions determine a measure of risk), the investment decisions on behalf of the potential risk factors represents a risk factor. $T$ weight position in the whole system of risk parameter $g$ represented It is the degree of uncertainty of various risk factors. At this stage, in accordance with the distinction between the sources of risk, risk factors can be divided. The existence of risk factors project itself, analytical bias risk factors of various external conditions of the project and feasibility study in particular artificial. On the stage of the project investment decisions, there is uncertainty over a variety of factors, it is not a random occurrence, but the uncertainty and ambiguity of the characteristics of the traditional decision-making assessment model, only able to determine, for the first time The model for analysis of quantitative reasoning, and in the process of decision, there are many man-made factors that exist, there is a big gap between the concrete result of the decision and the objective facts, thus establishing various determinants in the above formula is determined on the basis of on, to which the theory of fuzzy mathematics content, using fuzzy theory to do analysis and research projects, establish investment mathematical model of various outcomes do more reasonable, effective and practical analysis.

\section{Conclusion}

All in all, constructing mathematical model and mathematical knowledge can be used to solve practical problems and to promote economic progress, promote China's scientific and technological innovation. In the economic sphere, there are many examples and we should go on the cutting edge, step by step ahead. Therefore, scientific decision-making is very important, and it is essential, so how scientific decision? We can use mathematical method to analysis the problem encountered in the economic field and converted into mathematical problems and use mathematical methods to solve problems.

\section{References}

[1] Wang Ai. Probabilistic Mathematical Model in Investment Decision Making. Science and Technology Information, 2012, 09: 153-154.

[2] Wang Yan, Probability and Statistics Mathematical Model. The Investment Decision of Hebei North University (Natural Science), 2014, 04: 35-38.

[3] Shen Bo. Applied Probability and Statistics in Investment Decision. Management \& Technology (Head), 2014,10: 92-93. 